\title{
Couple et sexualité des femmes d'Afrique sub-saharienne vivant avec le VIH/sida en France
}

\author{
Dolorès POURETTE ${ }^{1}$
}

\begin{abstract}
Résumé
En France, l'épidémie de sida concerne une part croissante de femmes originaires d'Afrique sub-saharienne. À partir d'entretiens réalisés auprès de 32 femmes, cet article analyse l'impact du diagnostic sur les relations de couple, la sexualité et les projets reproductifs. Les femmes interviewées ayant des parcours biographiques et migratoires diversifiés, les répercussions de l'annonce de la séropositivité sont variées. Pour les jeunes femmes en couple, la découverte de la séropositivité peut être suivie d'une rupture conjugale et d'une démarche migratoire. Les recompositions conjugales sont alors principalement orientées par le devoir de protection du partenaire et de l'enfant à venir. Certaines font cependant l'expérience de situations de domination, notamment lorsque le nouveau partenaire est séronégatif. Les femmes plus âgées, ayant effectué une migration ancienne, vivent en couple séroconcordant, mais elles rencontrent des difficultés pour négocier l'utilisation du préservatif avec leur conjoint afin d'éviter une surcontamination.
\end{abstract}

Mots clés: migrantes, Afrique sub-saharienne, couple, sexualité, sérodifférence, désir d'enfant.

Les statistiques concernantl'épidémie de sida en France montrent que les étrangers, principalement originaires d'Afrique subsaharienne, représentent une part importante des diagnostics de séropositivité. En 2005, environ une découverte de séropositivité sur trois les concernait. La même année, la moitié des femmes dépistées venait d'un pays d'Afrique sub-saharienne [1].

La gravité de l'épidémie de sida en Afrique et les difficultés d'accès aux traitements antirétroviraux conduisent des femmes et des hommes à entreprendre des migrations vers l'Europe de l'Ouest où les conditions d'accès aux traitements et les possibilités de survivre avec le VIH sont nettement meilleures [2, 3]. Cependant, parmi les personnes originaires d'Afrique sub-saharienne vivant avec le VIH en France, certaines ont été dépistées pour la première fois en France. Et des analyses virologiques, soulignant la présence du sous-type $B$ (sous-type peu présent en Afrique) chez des patients originaires d'Afrique sub-saharienne, suggèrent que des contaminations ont lieu après l'arrivée en Europe [1].

Unité Inserm-Ined 822/Université Paris 5-Descartes. Recherche financée par Sidaction et Fondation de France.
Si depuis 1998, les étrangers peuvent prétendre au droit au séjour pour raison médicale 2 , le contexte législatif actuel va dans le sens d'un durcissement des critères et des procédures d'attribution de ce droit [4]. On observe, depuis 2003, une diminution marquée des taux d'accord dans les départements où la demande est importante [5] et des expulsions d'étrangers malades ont eu lieu au cours de l'année 2007. Par ailleurs, les demandeurs d'asile ne sont pas autorisés à exercer un emploi et une autorisation provisoire de séjour ne donne pas systématiquement droit au travail, ce qui renforce la vulnérabilité sociale des étrangers malades.

Longtemps occultée par la société française [6], la question du sida au sein des populations migrantes en France fait encore aujourd'hui l'objet d'un nombre restreint de recherches scientifiques. Cet article présente les résultats d'une étude qualitative menée auprès de migrantes ${ }^{3}$ originaires d'Afrique sub-saharienne vivant avec le VIH (cf encadré ci-après). II analyse l'impact de la découverte de la séropositivité sur les relations de couple et les recompositions matrimoniales, la sexualité et les projets reproductifs.

La population d'étude est hétérogène, au sens où elle regroupe desfemmes ayant des parcours biographiques, familiaux, sociaux, professionnels et migratoires variés. Cependant, les femmes enquêtées partagent la « condition » de malade et celle d'immigrée [9], et elles ont en commun d'appartenir à des catégories de genre et de " race " ${ }^{4}$ infériorisées : elles sont des " femmes", "noires ${ }^{5}$. Leur expérience du VIH et l'impact de la maladie sur les relations de couple et la sexualité sont appréhendés au regard de leur trajectoire migratoire, de l'histoire conjugale, de

\footnotetext{
2 Ce droit permet l'accès à un titre de séjour temporaire (un an) pour des personnes sans papiers atteintes d'affection grave et qui ne peuvent bénéficier dans leur pays d'origine de traitements appropriés.

3 En France, la désignation des populations migrantes, étrangères ou non, et de leurs descendants est particulièrement complexe et embarrassante [7]. Dans ce texte, les termes « migrants * et « migrantes * sont utilisés en référence à la définition des Nations Unies : « a migrant is defined as a person who has moved to a country other than that of their usual residence and has been living in that country for more than one year. The country of destination effectively becomes their new country of usual residence. $*[8, p .9]$.

4 Le terme * race * est utilisé au sens de produit d'un rapport social spécifique [10] et ne renvoie en aucun cas à une réalité biologique.

5 Ces deux catégories sont indiquées entre guillemets pour souligner qu'il ne s'agit pas de catégories naturelles, mais bien de catégories sociales, fruit d'un rapport social hiérarchisé.
} 


\section{Méthodologie de l'enquête}

32 femmes et 10 hommes ont été interviewés au cours d'entretiens semi-directifs individuels et anonymes, entre décembre 2004 et juillet 2006. Les enquêtés ont été recrutés dans plusieurs hôpitaux de la région parisienne et dans deux associations communautaires d'aide aux personnes vivant avec le VIH. Les critères de recrutement étaient les suivants : avoir plus de 18 ans, être né(e) dans un pays d'Afrique sub-saharienne, résider en France depuis un an au moins et avoir été diagnostiqué(e) séropositif(ive) au VIH depuis un an au minimum. Dans les hôpitaux, les médecins étaient chargés de présenter l'étude aux patients correspondant aux critères d'inclusion, et de leur demander leur accord pour y participer. Le même principe a été appliqué dans les associations. Le choix du lieu et du moment de l'entretien (à l'hôpital, dans un local associatif ou dans mon bureau) était laissé à la personne interviewée. Les entretiens, dont la durée allait de une à trois heures, étaient enregistrés avec l'accord de la personne enquêtée. Ils ont été transcrits puis analysés selon une perspective biographique et selon une perspective thématique transversale. Les femmes interviewées ont entre 22 ans et 51 ans (moyenne d'âge : 35 ans). Elles sont originaires de 11 pays (principalement la Côte d'Ivoire, le Congo-Brazzaville, la République démocratique du Congo, le Cameroun).

la situation sociale, incluant notamment le statut administratif, le rapport à l'emploi, les ressources, les réseaux sociaux.

\section{Des itinéraires biographiques variés}

Les trajectoires biographiques et migratoires des femmes interviewées sont très diversifiées. La majorité d'entre elles a été dépistée en France. Si quelques-unes y résidaient depuis plusieurs années, voire plusieurs dizaines d'années, la plupart étaient arrivées depuis peu (après 2000) et la découverte de leur séropositivité au VIH a été concomitante de leur démarche migratoire. Ces femmes, dont l'arrivée en France est récente, sont relativement jeunes (entre 22 ans et 45 ans, avec une moyenne d'âge de 33 ans), célibataires pour la plupart, et sans enfant ou dont les enfants, en bas âge, sont restés en Afrique. À l'inverse, les femmes qui ont appris leur contamination par le VIH après plusieurs années d'installation en Europe sont des femmes plus âgées (entre 27 ans et 51 ans, avec une moyenne de 40 ans), avec des enfants nés et vivant en France, mariées pour la plupart. Concernant les femmes qui ont été dépistées en Afrique, elles étaient mariées ou vivaient en couple dans leur grande majorité. La découverte de leur séropositivité a entrainé une révision des projets familiaux et professionnels et la mise en œuvre d'une stratégie migratoire dans un objectif d'accès aux soins.

Les femmes enquêtées ont en commun une précarité socioéconomique marquée au moment de l'entretien. Ainsi, sur 32 femmes, seules 5 ont un emploi à temps complet, 8 travaillent à temps partiel et 19 n'ont pas d'activité économique. La précarité économique et administrative des patients originaires d'Afrique sub-saharienne infectés par le VIH avait déjà été constatée en 2002, par une étude menée auprès de 250 patients suivis dans 19 hôpitaux franciliens [11].

\section{De l'absence de protection de soi à la nécessité de protéger les autres}

De nombreuses études ont mis en évidence les obstacles à l'adoption de comportements sexuels à moindre risque, y compris dans les pays très touchés par l'épidémie de sida, et ce même Iorsque la population est informée des risques de contamination $[12,13]$. En Afrique, ces obstacles sont notamment liés à l'image particulièrement dépréciée du préservatif, dont l'utilisation reste associée à l'idée de relations occasionnelles ou de peu d'importance, et qui est réputé amoindrir le plaisir [14, 15]. En outre, les femmes, du fait de l'inégalité des rapports entre les sexes, rencontrent certaines difficultés pour négocier la protection des relations sexuelles [14], bien que des études aient mis en évidence l'existence d'espaces de négociation $[14,16,17]$. Ainsi, les femmes enquêtées au cours de cette recherche témoignent d'une utilisation du préservatif très ponctuelle avant le diagnostic du VIH. Si quelques-unes avaient déjà utilisé un préservatif à l'occasion de relations nouvelles ou de passage, ou comme moyen de contraception, la majorité n'en avait jamais utilisé pour se prémunir du VIH ou d'une autre maladie sexuellement transmissible, même parmi celles qui résident en France de longue date (ces femmes se sont mariées au moment de leur arrivée en France, ou avant, et n'ont pas eu d'autres partenaires sexuels que leur conjoint). Comme dans d'autres contextes [13], c'est dans le cadre du couple que des comportements préventifs sont particulièrement difficiles à mettre en œuvre. Les femmes vivant en couple soulignent qu'avant de connaître leur séropositivité, il leur était très difficile de négocier la protection des relations, y compris lorsqu'elles étaient informées des infidélités de leur époux, ou lorsque la co-épouse de celui-ci était visiblement gravement malade 6 .

Alors qu'elles ne se protégeaient pas du VIH avant d'être dépistées, la protection de l'autre (le partenaire ou l'enfant à venir) devient l'une de leurs préoccupations principales après le diagnostic. Ce devoir de protection prime sur leur préservation d'un éventuel rejet social. Ainsi, doutant d'être en capacité d'assurer la protection du partenaire, en lui proposant le préservatif ou en évitant les rapports sexuels, et bien qu'elles redoutent les réactions de rejet, les femmes enquêtées informent majoritairement

\footnotetext{
L'une des femmes interviewées était engagée dans un mariage polygame. Elle a appris sa séropositivité après le décès de la co-épouse de son mari.
} 
leur partenaire des résultats du test (bien souvent très peu de temps après qu'elles soient elles-mêmes informées). Dire sa séropositivité au $\mathrm{VIH}$, au risque de la réprobation voire de la répudiation, apparaît ici comme un recours pour assurer la protection d'autrui. Cette parole est d'autant plus importante qu'elle prend place dans un contexte où la séropositivité a le statut de secret [18], parfois révélée à un ou quelques membres de la famille, mais rarement au-delà du cercle des intimes, comme cela a été souligné ailleurs $[19,20]$.

Les répercussions de cette parole sur la gestion du VIH au sein du couple ne sont pas les mêmes selon le moment de l'histoire conjugale où elle est énoncée : au début de la relation ou après plusieurs années de vie commune, alors que le couple vit en Afrique ou en France.

\section{Des couples face au VIH}

La moitié des femmes enquêtées vivaient en couple au moment du dépistage. La majorité était mariée, quelques-unes vivaient maritalement avec leur compagnon. Nous distinguerons les femmes qui résidaient en Afrique au moment du dépistage et celles qui étaient installées en France.

\section{Lorsque le VIH conduit à la rupture et à la migration}

Sept femmes vivaient en couple au moment où elles ont découvert leur séropositivité (quatre étaient mariées, dont deux qui avaient des enfants), trois vivaient avec leur compagnon sans être mariées (dont une qui avait un enfant d'une première union)).

Elles ont été diagnostiquées au moment d'une grossesse, d'une maladie ou d'un test volontaire. Seules deux femmes ont découvert leur séropositivité après l'annonce de celle du mari. Les autres ont informé leur conjoint et, bien que souvent redoutées, les réactions de rejet sont exceptionnelles. Elles ont cependant concerné deux femmes dont le conjoint aurait refusé le dépistage et mis fin à la relation.

Le plus souvent' ${ }^{7}$, l'annonce de la séropositivité a entraîné, à plus ou moins long terme (de quelques semaines à deux ans), la rupture conjugale. Les causes de ces ruptures sont multiples et complexes, mais les enquêtées évoquent deux éléments principaux : le fait que les conjoints se savaient déjà infectés et qu'ils ne les en avait pas informées (ce qui concerne trois femmes) ${ }^{8}$; le refus du conjoint d'envisager une migration en France en vue

\footnotetext{
Une femme est devenue veuve quelques mois après l'annonce de la séropositivité du couple. Une autre vit toujours avec son conjoint, en France, où ils se sont réfugiés suite à des conflits armés.

8 Pour les hommes comme pour les femmes, dévoiler sa séropositivité à son partenaire peut être très difficile, voire impossible. Ainsi, certains conjoints n'ont pas informé leur partenaire. Cette difficulté à partager les résultats du dépistage avec une conjointe a été exprimée par les hommes enquêtés au cours de cette étude : la crainte du rejet et du déshonneur de la part de l'épouse et, plus largement, du groupe social et la peur de ne pouvoir avoir de descendance sont souvent évoquées par des hommes qui ont le devoir d'assurer la reproduction économique et sociale du groupe familial.
}

d'accéder aux traitements antirétroviraux (deux femmes). Pour les premières, la séparation est directement liée à la révélation de la séropositivité dans le couple. Pour les autres, c'est davantage la dégradation de l'état de santé et les difficultés d'accès aux soins dans le pays d'origine qui ont motivé la migration et, par conséquent, la séparation d'avec le conjoint. La décision de partir n'est jamais facile à prendre, d'autant plus que cela signifie, pour les unes, de laisser des enfants à la famille ou à la bellefamille, pour d'autres, de renoncer à un mariage prévu de longue date, de quitter un homme aimé, ou d'abandonner des projets professionnels. Ces femmes ont cependant pu bénéficier d'un soutien pour organiser et mettre en œuvre leur départ : de la part de leur famille ${ }^{9}$, lorsqu'elle était informée, ou d'une association d'aide aux personnes infectées par le $\mathrm{VIH}$.

Les stratégies migratoires mises en œuvre dans ce contexte sont principalement motivées par la volonté de survivre et d'accéder aux traitements. Cependant, l'éloignement donne aussi la possibilité d'échapper au risque de réprobation qui peut concerner des femmes ayant rompu une relation conjugale, ainsi qu'aux rumeurs sur leur état de santé, les motifs incitant une personne à entreprendre une démarche migratoire pouvant être multiples $[8,21]$.

Si pour les femmes qui ont découvert leur séropositivité, alors qu'elles étaient engagées dans une relation conjugale et vivaient en Afrique, la découverte du VIH a le plus souvent été suivie d'une rupture conjugale, ce n'est pas le cas pour celles qui résidaient en France.

\section{Lorsque le VIH survient dans le pays d'immigration}

Les situations dont il est question ici concernent six femmes mariées ou vivant en couple de longue date, qui ont effectué une migration familiale dans les années 1980 et 1990 et qui sont installées en France depuis plus de dix ans. A la différence des femmes précédemment évoquées, elles ont une carte de résidente (dix ans) et quelques-unes ont la nationalité française. Pour la majorité, elles ont effectué leur migration avec leur époux. Elles ont entre 27 ans et 51 ans et ont de un à neuf enfants.

Le dépistage a eu lieu dans les années 1990, au moment d'une grossesse, d'une maladie ou d'une consultation pour stérilité. Une seule femme n'a pas informé son conjoint de sa séropositivité. Parmi les autres, trois ont appris que leur mari se savait déjà infecté, un époux refuse le dépistage, un autre a été diagnostiqué séropositif. Dans tous les cas, la nouvelle de l'infection dans le couple est arrivée par elles.

Afin de comprendre l'impact de l'annonce du VIH sur les relations de couple, il est nécessaire de prendre en compte les trajectoires migratoires et les situations sociales et familiales des femmes

\footnotetext{
Pour ne pas susciter d'inquiétude parmi les proches, les femmes évitent bien souvent de leur confier qu'elles sont séropositives. Celles qui l'ont fait n'ont pas rencontré de réaction de rejet, mais témoignent généralement d'un soutien affectif, si ce n'est financier.
} 
et de leurs époux. Ces couples font partie des générations d'immigrés pour lesquelles le travail masculin constituait le moteur de la migration. L'un des effets de ce type de migration est que les épouses n'ont pas été intégrées en France par l'emploi, mais davantage par leur rôle familial et reproductif. Ainsi, les femmes interviewées ne travaillent pas, ont peu de ressources personnelles, et sont économiquement dépendantes de leur mari. Par ailleurs, elles se trouvent relativement isolées en France : leurs familles vivent en Afrique et elles ne les ont pas informées de leur infection, secret partagé uniquement avec le conjoint. À la différence des femmes qui ont pu, dans leur pays, bénéficier d'un soutien familial sur le plan moral et matériel, et entreprendre une migration, ces femmes, dont l'implantation en France est plus ancienne, se trouvent davantage isolées par rapport à leur famille et elles ont développé peu de liens sociaux dans le pays d'immigration.

Par ailleurs, ces couples ont pour la plupart débuté leur vie sexuelle avant l'existence du $\mathrm{VIH}$, et ont une expérience commune inexistante ou limitée du préservatif. Ils semblent avoir été peu informés sur les risques de contamination au sein du couple - les campagnes de prévention en France n'ayant pas mis l'accent sur cet aspect et ne s'adressant aux populations migrantes que depuis une période récente. De plus, ils ne fréquentent pas d'association communautaire (ni avant, ni après l'annonce de la maladie), comme les associations de soutien aux personnes vivant avec le VIH, où ils pourraient être informés des risques de surcontamination dans un couple concordant par exemple.

Comme de nombreuses études l'ont montré, les personnes qui n'ont jamais utilisé le préservatif, et qui ont débuté leur vie sexuelle avant l'existence du sida, rencontrent plus de difficultés que les autres pour modifier leurs comportements sexuels après la découverte d'une séropositivité $[12,22]$. Ainsi, les discours des femmes enquêtées montrent que l'annonce de la séropositivité n'est pas systématiquement suivie d'une modification des pratiques sexuelles, ni même d'un dialogue au sein du couple à propos de la contamination, de son origine, et d'éventuels ajustements de la vie sexuelle et reproductive. Pour certains couples, le VIH a une réalité médicale (qui se manifeste au moment des consultations et de la prise des traitements), mais il ne semble pas avoir de répercussions sur les rapports conjugaux. Ainsi, dans le discours de Ténemba ${ }^{10}$, l'hypothèse d'une contamination sexuelle n'est pas évoquée -, elle suppose avoir été contaminée au cours de soins médicaux reçus dans sa jeunesse. De même, l'éventualité d'une surcontamination au sein du couple n'est pas suggérée, ni celle d'une transmission maternofœtale (Ténemba ayant eu une ligature des trompes après son dernier accouchement).

D'autres femmes témoignent d'une volonté de se protéger et de protéger leur conjoint d'une éventuelle surcontamination. Elles

\footnotetext{
1046 ans, née au Mali, en France avec son mari (employè dans une entreprise de ménage industriel) depuis 27 ans, neuf enfants.
}

rencontrent néanmoins des difficultés pour aborder avec celui-ci la possibilité d'une modification des pratiques sexuelles et, notamment, l'adoption du préservatif. Des espaces de négociation sont cependant évoqués : ainsi, Tissina ${ }^{11}$ affirme parvenir à espacer les rapports sexuels, en les refusant de temps en temps. Mais elle ne peut pas s'y soustraire totalement. L'enjeu de la protection se double ici d'un enjeu de procréation : elle "doit » faire un troisième enfant. Après être "restée quatre ans sans faire d'enfants ", " tout autour, la famille, la mère parle : normalement c'est trois minimum ».

Lorsque l'époux refuse le dépistage, en arguant par exemple que le sida "n'existe pas", la négociation des rapports sexuels et de leur protection s'avère très difficile. Ainsi, Mariama ${ }^{12}$ ne parvient pas à instaurer un dialogue avec son mari sur sa maladie, sur leur vie sexuelle et reproductive, sur le fait qu'il peut aussi être contaminé. Elle ne sait pas s'il s'est fait dépister, bien qu'elle suppose qu'il est également infecté. En outre, si son mari a toujours été violent avec elle, il l'est davantage encore depuis qu'elle a été dépistée. II l'oblige notamment à avoir des rapports sexuels avec lui, sans préservatif, afin d'avoir une descendance. II a menacé Mariama de prendre une seconde épouse si elle ne mettait pas au monde un enfant. Elle s'est donc sentie "obligée " d'avoir un enfant en dépit de sa peur qu'il soit infecté ${ }^{13}$. Si elle avait refusé cette grossesse, elle se serait sentie responsable de la contamination éventuelle d'une autre femme.

Finalement, parmi les femmes de cette sous-catégorie, la seule qui parvient à utiliser le préservatif avec son conjoint est celle qui ne l'a pas informé de sa séropositivité. Pauline ${ }^{14}$ a découvert son infection au cours d'examens médicaux pratiqués car elle ne parvenait pas à être enceinte, après avoir eu trois enfants avec son mari. Lui étant très attachée, elle redoute que la nouvelle de sa séropositivité nuise à leur relation. Cependant, depuis qu'elle connaît son statut sérologique, Pauline fait en sorte que son époux utilise systématiquement des préservatifs. En effet, elle utilise l'argument d'un traitement contre sa stérilité ${ }^{15}$ comme moyen de prévention: ce traitement contre-indiquerait une grossesse actuellement, grossesse qui pourra être envisagée au terme de la thérapie. Le mari de Pauline ne semble pas réticent à l'utilisation du préservatif, d'autant plus qu'il a l'habitude de l'utiliser et que le couple y avait recours au début de la relation et au moment du post-partum. Elle envisage d'amener son époux à se faire dépister au moment où elle décidera de tenter une nouvelle grossesse, sans l'informer au préalable de sa séropositivité.

Intéressons-nous maintenant aux femmes qui ne vivaient pas en couple au moment du diagnostic.

\footnotetext{
35 ans, nèe au Congo, en France depuis 1987. Mariée et mère de deux enfants, elle a découvert sa séropositivité au cours de sa deuxième grossesse, en 1999.

1230 ans, née au Sénégal, en France depuis l'âge de 18 ans. Mariée une première fois, elle a eu un fils, puis a divorcé et s'est remariée. Dépistée en 1999, à la suite d'une maladie. 13 L'enfant est séronégatif.

1432 ans, née en Côte d'lvoire, mariẻe, trois enfants. Réside en France depuis 1998.

15 En réalité, elle est sous thérapies antirétrovirales pour le VIH.
} 


\section{Des relations de couple asymétriques}

Au moment où elles ont été dépistées, quelques femmes avaient débuté une relation amoureuse avec de nouveaux partenaires. Certaines n'avaient pas encore eu de rapports sexuels avec eux, d'autres en avaient, mais toujours en utilisant le préservatif. Le test de dépistage a été pratiqué par l'homme et par la femme avant d'avoir des relations sexuelles ensemble ou dans le but d'arrêter le préservatif. Dans tous les cas, l'homme est séronégatif. Ces couples, sérodifférents, ont décidé de poursuivre la relation, puis de se marier.

Les partenaires de ces femmes sont tous des hommes français, "blancs "16, qu'elles ont rencontrés en Afrique avant de venir s'installer avec eux en Europe, ou en France peu après leur arrivée. Ces relations conjugales s'inscrivent dans des relations de pouvoir asymétriques, du fait de la sérodifférence et du fait des appartenances socioraciales des deux acteurs.

Pour ces femmes, un mariage avec un Français ou le fait d'avoir un enfant reconnu de père français constitue souvent le seul moyen d'obtenir des papiers [24]. Si elles n'évoquent pas cette dimension au cours des entretiens, elles expriment combien elles se sentent redevables de leurs conjoints, qui ont accepté de poursuivre une relation avec une femme séropositive et d'envisager une descendance avec elles, qui leur ont permis d'accéder à la santé, et à une formation professionnelle ou à un niveau de vie bien supérieur à celui que leurs propres capitaux sociaux et scolaires auraient pu leur offrir. Elles accèdent ainsi à une certaine reconnaissance sociale dans le pays d'immigration, mais également auprès du groupe social d'origine, qui bénéficie indirectement (sous forme de prestige) et directement (sous forme d'aide financièr ${ }^{17}$ ) de leur promotion sociale.

Ces femmes se perçoivent néanmoins en position de débitrices, ce qui les amène à faire certaines concessions, au quotidien, comme d'admettre des remarques et des comportements blessants ou dévalorisants. Dans quelques cas, ces situations de domination se traduisent par des violences, psychologiques et morales (interdiction de sortir, de travailler, brimades), physiques et sexuelles ${ }^{18}$. Nous retrouvons ainsi le lien établi par d'autres études entre la séropositivité et l'exposition aux violences du conjoint. S'il a été montré que le fait de subir des violences influe négativement sur les possibilités pour la femme de négocier les rapports sexuels et leur protection [25-27], le risque de subir des

\footnotetext{
16 L'utilisation du lexique de la couleur s'explique par l'articulation des questions sociales et des questions raciales, la couleur étant le signe d'un rapport social inégalitaire et s'articulant à la condition sociale et, notamment, à la nationalité [23]. II va de soi que tous les hommes « blancs * n'ont pas la nationalité française, mais lorsque les femmes enquêtées utilisent les termes « blancs * ou " européens ", ces termes désignent des personnes françaises, ce qui n'est pas nécessairement le cas des personnes qu'elles désignent comme " africaines *.

17 L'une des femmes interviewées explique par exemple que l'ensemble de son village vit grâce à ses revenus et à la position sociale de son mari.

18 Françoise Guillemaut fait une observation similaire auprès de travailleuses du sexe migrantes, dont certaines, mariées ou vivant en concubinage, subissent des violences conjugales [24].
}

violences est plus important pour les femmes infectées que pour les femmes non infectées [28, 29], les violences pouvant être l'une des conséquences de la révélation du statut sérologique au conjoint [30, 31].

Les femmes concernées expriment leurs difficultés à résister à ces violences, car elles craignent que leur mari ne mette fin à la relation. On mesure ici « la pression que peut exercer le cadre juridique sur les femmes " ([24, p. 111], une femme étrangère qui divorce risquant de perdre son titre de séjour. La crainte de la perte des papiers se double pour les femmes vivant avec le VIH de la peur de perdre également l'accès aux soins. De surcroît, certains conjoints utilisent la menace de dévoiler à l'entourage la séropositivité des femmes concernées, si elles se montrent récalcitrantes ou si elles envisagent de les quitter.

Sur le plan sexuel, les rapports sont généralement protégés, sauf dans quelques circonstances précises ${ }^{19}$ : la protection est parfois momentanément suspendue, d'un commun accord, en vue d'une procréation ${ }^{20}$. Cependant, même après plusieurs années d'union et même lorsque les rapports sont protégés, la crainte de contaminer le partenaire est toujours présente ${ }^{21}$. Une femme rapporte que son conjoint s'est toujours opposé à l'utilisation du préservatif. Au cours des dix années qu'a duré leur relation conjugale, elle n'est pas parvenue à ce qu'il se protège d'une infection éventuelle. Elle décrit une expérience douloureuse de la sexualité conjugale, qu'elle dépeint comme angoissante, culpabilisante et marquée par la crainte de le contaminer.

\section{Des recompositions matrimoniales dans le contexte du VIH}

Si les contextes sociaux, politiques, économiques sont structurellement contraignants et marqués par des inégalités liées au genre, à la « race », à la classe, et s'ils limitent les possibilités des femmes, il n'en demeure pas moins que celles-ci ne subissent pas passivement leur situation et opèrent certains choix pouvant leur permettre d'améliorer leur situation $[8,36]$. Par exemple, la migration, y compris par le biais de filières informelles, peut constituer une stratégie visant à se soustraire à des inégalités [37].

Dans leurs parcours matrimoniaux, les femmes interviewées opèrent des choix, en fonction de la perception qu'elles ont de leur situation actuelle, de leurs perspectives d'avenir, des possibilités qui s'offrent à elles, mais également en fonction de

\footnotetext{
19 Les résultats de l'enquête Vespa montrent que les couples hétérosexuels sérodifférents rapportent des relations non protégées ( $26 \%$ des hommes et $33 \%$ des femmes), qu'il s'agisse de migrants ou non. Cependant, les femmes immigrées, principalement originaires d'Afrique sub-saharienne, rapportent moins de pratiques à risque dans le cadre de couples sérodifférents [32].

20 Janine Pierret fait la même observation pour les personnes qu'elle a interviewées en France [20].

21 Les recherches auprès de personnes infectées par le VIH soulignent ces difficultés dans la vie sexuelle après le dépistage [20,33-35].
} 
leurs représentations des hommes, selon leur origine, leur statut sérologique et en fonction de la manière dont elles pensent être perçues par ces hommes (en tant que femmes noires, immigrés, étrangères, séropositives).

Comme de nombreuses études l'ont montré, le diagnostic du VIH est très souvent suivi d'une période d'absence de désir sexuel et de suspension momentanée des projets de vie (familiaux, professionnels...) $[20,34]$. Les femmes célibataires au moment du diagnostic, ou qui le sont devenues par la suite, témoignent de cette interruption plus ou moins temporaire des perspectives d'avenir et elles n'envisagent pas, au moins dans un premier temps, de renouer des relations affectives. La difficulté à dire à un nouveau partenaire que l'on est infecté par le VIH, la crainte du rejet et la peur de contaminer l'autre mènent à l'abstinence, affective et sexuelle. Si quelques femmes ayant déjà été mariées et ayant des enfants en âge d'être eux-mêmes parents ont décidé de rester seules de manière définitive, pour les autres, l'acquisition d'un "savoir sur le sida" [38], par les contacts avec le milieu médical et parfois le milieu associatif, et la prise de conscience que l'on peut vivre avec le VIH et donner naissance à des enfants non infectés, mènent à élaborer de nouveaux projets et à envisager de nouvelles relations affectives. L'analyse des trajectoires conjugales souligne que les stratégies matrimoniales qui sont mises en œuvre dans le contexte de la séropositivité sont orientées par une double contrainte : d'une part, le partenaire doit être susceptible d'accepter d'être en couple avec une femme séropositive et de mener un projet d'enfant avec elle ; d'autre part, les risques de contamination (du partenaire et de l'enfant à venir) doivent être limités.

Une première stratégie matrimoniale concerne des femmes qui ont noué des relations (ou envisagent de le faire) avec des hommes originaires d'Afrique sub-saharienne également infectés par le VIH. II s'agit de femmes dont l'expérience de la migration et celle du VIH sont imbriquées : elles ont migré pour accéder aux traitements, ou elles ont découvert leur séropositivité peu de temps après leur arrivée. Ces femmes sont impliquées dans des réseaux de sociabilité liés à la maladie (hôpitaux, associations) et, en dehors de ces réseaux, elles ont développé peu de liens avec la population non immigrée. Elles fréquentent régulièrement les associations communautaires d'aide aux personnes séropositives où elles rencontrent des hommes et des femmes se trouvant dans des situations similaires au regard de la maladie et de la migration. Pour ces femmes, une relation avec une personne immigrée et également séropositive représente le moyen de se sentir comprises et soutenues par une personne qui partage les mêmes difficultés. Ces femmes sont aussi celles qui supposent qu'aucun homme séronégatif n'accepterait de vivre avec une femme séropositive. En orientant leur choix vers des hommes séropositifs uniquement, elles évitent de s'exposer à une réaction de rejet ou à un regard dépréciatif lors de l'annonce de leur infection.
Une deuxième stratégie matrimoniale concerne des femmes pour lesquelles seule une union avec un "Européen " est envisageable. Ces femmes vivent en France depuis plus longtemps que les femmes précédemment évoquées, et elles ont développé des liens de sociabilité au sein des populations migrantes et non migrantes. Elles justifient leur orientation par une représentation des hommes " européens " comme étant susceptibles d'accepter de vivre avec une femme séropositive, et avec lesquels il leur sera plus facile d'établir un dialogue sur la sexualité et le sida et de négocier l'utilisation du préservatif. Cette perception fait parfois suite à des expériences décevantes auprès d'hommes qu'elles qualifient d'، Africains" et qui auraient interrompu la relation après qu'elles les aient informés de leur séropositivité. À l'instar de migrantes résidant en Suisse, ces femmes présentent une vision idéalisée des hommes " européens ", qui oriente leurs stratégies matrimoniales vers une recherche de relations plus égalitaires [39]. Nous avons cependant souligné la dimension asymétrique de ces relations.

Au cœur de ces stratégies matrimoniales, les projets reproductifs tiennent une place importante.

\section{Le désir d'enfant : entre statut social et gestion du risque de transmission}

Les recherches menées dans les pays d'Afrique ont souligné l'importance accordée à la maternité comme élément constitutif de l'identité féminine $[40,41]$. Malgré le risque de transmission maternofœtale qui demeure élevé dans les pays d'Afrique sub-saharienne, et en dépit des conseils de prévention qui sont délivrées aux femmes séropositives, leur fécondité reste élevée après le diagnostic [42-44]. Ainsi, en Côte d'Ivoire, le désir d'enfant demeure important après le dépistage chez la majorité des femmes, à l'exception de celles qui estiment avoir eu suffisamment d'enfants [13]. En France, parmi les femmes originaires d'Afrique sub-saharienne, l'incidence des grossesses augmente après le diagnostic du VIH chez les femmes jeunes et ayant moins de deux enfants [45], et le désir d'enfant, chez les femmes et chez les hommes, est plus marqué que parmi les personnes nées en France [46].

Les femmes interviewées soulignent également combien il est important pour elles d'être mères, et combien le diagnostic du VIH a mis en question leur capacité à enfanter. Elles sont informées du fait que les traitements antirétroviraux réduisent considérablement le risque qu'une femme séropositive donne naissance à un enfant infecté. Si le fait de vivre en France signifie l'accès aux traitements médicaux et à la santé, il donne également l'accès à la maternité. Seules les femmes les plus âgées, qui ont déjà enfanté et dont les aînés sont en âge de procréer ne souhaitent plus avoir d'enfant. Les plus préoccupées par les projets reproductifs sont les femmes les plus jeunes, qui n'ont pas d'enfant ou qui ont dû laisser les leurs en Afrique. 
Si quelques femmes évoquent les pressions exercées par le conjoint ${ }^{22}$, la belle-famille ou la famille pour qu'elles donnent naissance à un premier enfant ou à un autre enfant, la majorité exprime surtout un besoin personnel d'être mère. Alors qu'elles ont perçu le diagnostic du VIH comme l'annonce d'une mort proche, comme la suspension de toute perspective d'avenir et comme la mise en question de leur capacité à enfanter, avoir un enfant représente pour elles un moyen de se réaliser en tant que femmes et également de demeurer " vivantes ", à travers l'enfant, après la mort. La maternité reste donc un moyen d'acquérir un statut social valorisant dans un contexte de dépréciation sociale et personnelle (maladie, déclassement professionnel ou retrait de l'emploi...) Avoir un enfant en France, éventuellement de père français, peut aussi apparaître comme un moyen d'obtenir des papiers et comme l'assurance de pouvoir rester en Europe à long terme.

Comme pour les femmes séropositives résidant à Abidjan, l'enfant à venir apparaît ainsi comme un « gage de survie " pour l'individu " dans sa descendance " [47]. Mais cet aspect prend une dimension particulièrement importante dans la migration et dans un nouvel espace social, où cette descendance doit s'inscrire et prendre racine. En effet, le désir de donner naissance à un enfant en France est exprimé par plusieurs femmes : il s'agit pour elles de projeter leur avenir dans l'espace migratoire et de construire des liens sociaux et familiaux dans ce nouveau contexte de vie.

Si la majorité des femmes interviewées dit vouloir un enfant, seule une minorité d'entre elles a enfanté depuis l'annonce de sa séropositivité. Et celles qui envisagent une grossesse soulignent combien ce projet est réfléchi, mûri et discuté avec le conjoint, mais également avec le médecin et éventuellement d'autres acteurs (soignants, membres d'associations, femmes séropositives ayant enfanté). Ainsi, les projets reproductifs sont pensés en fonction des risques de santé, pour la mère et l'enfant. Le plus souvent, la grossesse n'est concrètement envisagée que lorsque les risques d'infection maternofœtale sont limités et lorsque l'état de santé de la mère le permet. Cette observation souligne l'importance de la parole et des conseils délivrés par le médecin. Elle invite également à rompre, comme certains auteurs l'ont fait, avec une vision culturaliste des malades immigrés comme des malades non "observants " des traitements médicaux et des conseils délivrés par les soignants [48-50]. Ainsi, même les femmes pour lesquelles l'injonction familiale à la maternité est forte parviennent à différer les grossesses de manière à être enceintes au moment où le médecin le juge opportun d'un point de vue médical. Tissina (déjà évoquée plus haut) attend ainsi « le feu vert » de son docteur pour suspendre son mode de contraception et démarrer une grossesse.

\footnotetext{
22 Les entretiens menés avec des hommes dans le cadre de cette recherche soulignent que le désir d'enfant est également très important chez les hommes séropositifs, dimension généralement peu prise en compte dans les études auprès des personnes vivant avec le VIH [46].
}

\section{Conclusion}

Les trajectoires sociales, affectives, reproductives et l'expérience de la sexualité dans le contexte de la migration et du $\mathrm{VIH} / \mathrm{sida}$ s'inscrivent dans des contextes sociaux et politiques, qui déterminent les conditions d'accès aux soins, à la citoyenneté et à l'emploi. La nécessité de rester en France pour pouvoir se soigner, les difficultés rencontrées pour obtenir un titre de séjour, une autorisation de travail et, le cas échéant, un emploi permettant de vivre en France, conduisent des femmes à s'engager dans des unions pouvant être inégalitaires. Outre le fait que l'accès à l'emploi est conditionné par l'obtention d'une autorisation de travail, donc d'un permis de séjour y donnant droit, les migrantes doivent faire face à un marché de l'emploi fondé sur le genre et sur la race [8]. En effet, quel que soit le niveau de qualification et la profession exercée dans le pays d'origine, les opportunités d'emploi sont restreintes à des activités de services domestiques traditionnellement associées au rôle assigné aux femmes dans la sphère privée et généralement à temps partiel [51]. Des femmes construites comme " africaines " n'étant pas perçues a priori comme des femmes diplômées ${ }^{23}$, les rares emplois qui sont proposés à celles qui ont une autorisation de travailler relèvent d'activités domestiques peu valorisées et peu rémunératrices (garde d'enfants ou de personnes âgées, ménage), ce qui renforce leur vulnérabilité sociale [52].

Dans le domaine de la sexualité, la principale stratégie que les femmes enquêtées mettent en œuvre pour protéger leurs partenaires et les enfants à venir repose sur le partage du secret de leur séropositivité. Cette parole est loin de garantir l'adoption de mesures préventives. En effet, les interviewées ne semblent pas rencontrer de difficultés pour négocier l'utilisation du préservatif avec des partenaires de rencontre, qui ne sont pas informés de leur statut sérologique, la norme préventive étant de mise dans ce type de relations sans enjeu de parentalité ni de conjugalité. À l'inverse, c'est au sein du couple, que celui-ci soit séroconcordant ou sérodifférent, avec des partenaires informés de leur séropositivité, qu'elles rencontrent le plus de difficultés pour négocier l'utilisation du préservatif. Cela souligne la nécessité de prodiguer des conseils de prévention auprès des deux membres du couple, sérodifférent ou séroconcordant, pour limiter les risques de contamination ou de surcontamination, comme cela a été mis en évidence dans d'autres contextes [53,54].

Les quelques femmes qui n'ont pas informé leur partenaire de leur séropositivité détiennent une certaine marge de manœuvre pour le protéger d'une éventuelle contamination : en invoquant des contre-indications médicales à la prise d'un contraceptif oral ou à la mise en route d'une grossesse. Ainsi, comme des recherches l'ont souligné en Côte-d'Ivoire, où des femmes retardent la reprise des rapports sexuels après une naissance pour protéger leur conjoint [55], c'est autour de la maternité

\footnotetext{
3 L'une d'elles a pourtant un DEA en histoire.
} 
que des marges de négociation sont mobilisées par les femmes séropositives désirant protéger leur partenaire d'une éventuelle contamination.

\section{Remerciements}

Je remercie Nathalie Bajos et l'unité Inserm-Ined 822 pour l'encadrement scientifique de cette étude, ainsi que Sidaction et la Fondation de France qui l'ont financée.

\section{Références bibliographiques}

1. Institut de Veille Sanitaire. Surveillance de l'infection à VIH-sida en France, 2005. Bulletin épidémiologique hebdomadaire 2006 ; 48:371-8.

2. Prost A. A review of research among black African communities affected by HIV in the UK and Europe. MRC Social and Public Health Science Unit. Occasional Paper no. 15. University of Glasgow, 2006.

3. Smacchia C, Di Perri G, Boschini A et al. Immigration, HIV infection, and sexually transmitted diseases in Europe. AIDS Patients Care \& STDs $2000 ; 14$ (5) : 233-7.

4. Sopena A. Nouvelle stratégie contre les malades étrangers. Plein Droit 2006 ; $71: 26-29$.

5. Veïsse A. Le médecin, la santé et le séjour des étrangers. Plein Droit 2006 ; $69: 32-35$

6. Fassin D. L'altérité de l'épidémie. Les politiques du sida à l'épreuve de I'immigration. Revue Européenne des Migrations Internationales 2001 ; 17 (2) : 139-151.

7. Fassin D. Nommer, interpréter. Le sens commun de la dénomination raciale. In De la question sociale à la question raciale. Représenter la société française, D Fassin et E Fassin, Eds. Paris : La Découverte, 2006, p. 19-36.

8. Kofman E, Phizacklea A, Raghuram P et al. Gender and International Migration in Europe. Employment, welfare and politics. London and New York : Routledge, 2000.

9. Fassin D. Une double peine. La condition sociale des immigrés malades du sida. L'Homme $2001 ; 160$ : 137-162.

10. Guillaumin C. L'idéologie raciste. Genèse et langage actuel. Paris : Gallimard, 2002 [1972].

11. Valin N, Lot F, Larsen C et al. Parcours sociomédical des personnes originaires d'Afrique sub-saharienne atteintes par le VIH, prises en charge dans les hôpitaux d'Île-de-France, 2002. Paris : InVS, 2004.

12. Bajos $N$. Les risques de la sexualité. In La sexualité aux temps du sida, In : N Bajos, M Bozon, A Ferrand et al, Eds. Paris : PUF, 1998, p. 35-61.

13. Desgrées du Lô̂ A. Le couple face au VIH/sida en Afrique sub-saharienne: information du partenaire, sexualité et procréation. Population $2005 ; 60$ (3) : 221-242.

14. Vidal L. Femmes en temps de sida : expériences d'Afrique, Paris : PUF, 2000.

15. Desclaux A et Desgrées du Loû $A$. Les femmes africaines face à l'épidémie de sida. Population et Sociétés $2006 ; 428: 1-4$.

16. Yana S D. Statuts et rôles féminins au Cameroun. Réalités d'hier, images d'aujourd'hui. Politique Africaine 1997 ; 65 : 35-47.

17. Desgrées du Loû A, Msellati P, Viho I et al. Contraceptive use, protected sexual intercourse and incidence of pregnancies among African HIV-infected women.
Ditrame Anrs 049 Project, Abidjan, 1995-2000. Int J of STD and AIDS 2002 ; $13(7): 462-8$

18. Zempléni A. Savoir taire. Du secret et de l'intrusion dans la vie des autres. Gradhiva $1996 ; 20: 23-43$.

19. Doyal L et Anderson J. « My fear is to fall in love again... " How HIV-positive African women survive in London. Soc Sci Med 2005 ; 60 : 1729-38.

20. Pierret J. Vivre avec le VIH. Enquête de longue durée auprès des personnes infectées. Paris : PUF, 2006.

21. Morokvasic M. Women in migration. In One Way Ticket, A Phizacklea, Ed. London : Routledge, 1983, p. 13-31.

22. Beltzer $\mathrm{N}$, Lagarde $\mathrm{M}$, Wu-Zhou $\mathrm{X}$ et al. Les connaissances, attitudes, croyances et comportements face au VIH/sida en France. Evolutions 1992 1994-1998-2001-2004. Paris : Anrs-ORS-Inpes, 2005.

23. Fassin D et Fassin E. De la question sociale à la question raciale. Représenter la société française. Paris : La Découverte, 2006.

24. Guillemaut $\mathrm{F}$ (dir.). Femmes et migrations en Europe. Stratégies et empowerment. Lyon : Le Dragon Lune Editions, 2004.

25. Van der Straten A, King R, Grinstead 0 et al. Couple communication, sexual coercion and HIV risk reduction in Kigali, Rwanda. AIDS $1995 ; 9$ (8) : 935-44.

26. Jewkes RK, Levin JB, Loveday A et al. Gender inequalities, intimate partner violence and HIV preventive practices: findings of a South African crosssectional study. Soc Sci Med $2003 ; 56: 125-134$.

27. Murray LK, Haworth A, Semrau K et al. Violence and abuse among HIV-infected women and their children in Zambia : a qualitative study. J Nerv Ment Dis $2006 ; 194(8): 610-5$

28. Maman S, Campbell J, Sweat MD et al. The intersections of HIV and violence: directions for future research and interventions. Soc Sci Med $2000 ; 50(4)$ : 459-78.

29. Maman S, Mbwambo JK, Hogan NM et al. HIV-positive women report more lifetime partner violence : findings from a voluntary counseling and testing clinic in Dar es Salaam, Tanzania. Am J Public Health 2002; 92(8):1331-7.

30. Gielen AC, O'Campo P, Faden RR et al. Women's disclosure of HIV status: experiences of mistreatment and violence in an urban setting. Women Health $1997 ; 25(3): 19-31$

31. Gielen AC, McDonnell KA, Burke JG et al. Women's lives after an HIV-positive diagnosis : disclosure and violence. Matern child Health J. $2000 ; 4(2)$ : 111. 20.

32. Bouhnik A D, Préau M, Lert $F$ et al. Unsafe sex in regular partnerships among heterosexual persons living with HIV : evidence from a large representative sample of individuals attending outpatients services in France (Anrs-Vespa Study). AIDS $2007 ; 21$ Supplement $1: 57-62$.

33. Green G. Sex, love and seropositivity. In Safety, sexuality and risk, P Aggleton, P Davies, G Hart, Eds. London : Taylor and Francis, 1995: 144-158.

34. Delor F. Séropositifs : trajectoires identitaires et rencontres du risque. Paris: L'Harmattan, 1997.

35. Pierret J. Vie sexuelle et affective des personnes asymptomatiques de longue durée. In : Anrs, Séropositivité, vie sexuelle et risque de transmission du VIH. Paris : Anrs, 1999, p. 71-83

36. Goss $\mathrm{J}$ et Lindquist $\mathrm{B}$. Conceptualizing international labor migration: a structuration perpective. International Migration Review $1995 ; 29$ (2) : 31751.

37. Moujoud N et Pourette D. "Traite * de femmes migrantes, domesticité et prostitution. A propos de migrations interne et externe. Cahiers d'Etudes Africaines 2005; 179-180: 1093-1121. 
38. Vidal L. Ritualités, santé et sida en Afrique. Pour une anthropologie du singulier. Paris : Karthala, 2004.

39. Riaño $\mathrm{Y}$ et Baghdadi $\mathrm{N}$. Je pensais que je pourrais avoir une relation plus égalitaire avec un Européen. Nouvelles questions féministes $2007 ; 26$ (1) : 38-53.

40. Desgrées du LoûA. Santé de la reproduction et sida en Afrique sub-saharienne. Enjeux et défis. Population $1998 ; 4: 701-730$.

41. Héritier F. Stérilité, aridité, sécheresse. Quelques invariants de la pensée symbolique. In Le sens du mal. Anthropologie, histoire, sociologie de la maladie, M Augé, C Herzlich, Eds. Paris : Editions des Archives contemporaines, 1984, p. 123-154.

42. Allen S, Serufilira A, Gruber, V, et al. Pregnancy and contraception use among urban rwandan women after HIV testing and counselling. Am J Public Health 1993 ; 83 (5) : 705-710.

43. Keogh P, Allen S, Almedal C et al. The social impact of HIV infection on women in Kigali, Rwanda : A prospective study. Soc Sci Med 1994 ; 38 (8) : 1047-53.

44. Nebié Y, Meda N, Leroy V et al. Sexual and reproductive life of women informed of their seropositivity : a prospective cohorte study in Burkina Faso. J Acquir Immune Defic Syndr $2001 ; 28$ (4) : 367-372.

45. Fourquet F, Le Chenadec J, Mayaux MJ et al. Reproductive behaviour of HIV-infected women living in France, according to geographical origin. AIDS $2001 ; 15$ (16) : 2193-2196.

46. Heard I, Sitta R, Lert F et l'équipe Vespa. Reproductive choice in men and women living with HIV : evidence from a large representative sample of outpatients attending French hospitals (Anrs-Vespa Study). AIDS 2007; 21 Supplement $1: 77-82$.
47. Desgrées du Loû A. Procréation et allaitement : quels choix pour une femme séropositive à Abidjan ? In Allaitement et VIH en Afrique de l'Ouest, A Desclaux, B Taverne, Eds. Paris : Karthala, $2000: 385-407$.

48. Fassin D et Ricard E. Les immigrés et le sida : une question mal posée. In Sida et vie psychique. Approche clinique et prise en charge, S Hefez, Ed. Paris : La Découverte, 1996 : 81-90.

49. Vidal L. Les pièges du culturalisme. Transcriptase $2004 ; 114: 29-31$.

50. Bonnet D. Drépanocytose et ethnicité. In La drépanocytose. Regards croisés sur une maladie orpheline, A Lainé, Ed. Paris : Karthala, 2004, p. 45-73.

51. Ferreira de Macêdo MB. Femmes de ménage et veilleurs de nuit: une approche sexuée du travail précaire dans un hôtel en France. Cahiers du Genre $2003 ; 35: 189-208$.

52. Pourette D. « Migratory Paths, Experiences of AIDS, and Sexuality : African Women Living with HIV in France ». Feminist Economics à paraitre en 2008.

53. Allen S, Meinzen-Derr J, Kautzman $M$ et al. Sexual behavior of HIV discordant couples after HIV counseling and testing. AIDS 2003; 17 (5) : 733-40.

54. Jones DL, Ross D, Weiss SM et al. Influence of Partner Participation on Sexual Risk Behavior Reduction Among HIV-Positive Zambian Women. Journal of Urban Health: Bulletin of the New York Academy of Medicine 2005; 82 (3 suppl 4) : 92-100.

55. Desgrées du Loû $A$ et Brou H. La reprise des relations sexuelles après une naissance : normes, pratiques et négociations à Abidjan, Côte d'Ivoire. LPED UMR 151, IRD-Université de Provence. Documents de recherche $n^{\circ} 3$. Série Santé de la reproduction, fécondité et développement, 2004. 